Pacific Journal of Mathematics

AN INEQUALITY FOR CLOSED SPACE CURVES

January 1962 


\section{AN INEQUALITY FOR CLOSED SPACE CURVES}

\section{G. D. ChaKerian}

1. Among a number of interesting results in a paper of I. Fáry (see [2]) appears the following. Let $C$ be a rectifiable closed curve of length $L(C)$ and total curvature $\kappa(C)$ enclosed by a sphere $S$ of radius $r$ in Euclidean 3-space. Then

$$
L(C) \leqq \frac{4}{\pi} r \kappa(C) .
$$

The proof of (1) rests upon the corresponding inequality for plane closed curves, which states that if $C$ is enclosed by a circle of radius $r$, then

$$
L(C) \leqq r \kappa(C) .
$$

The latter inequality gives a sharp result, with equality obtained in case $C$ is a circle of radius $r$.

In this paper we sharpen (1) to the following result. Let $C$ be a rectifiable closed curve enclosed by a $k-1$ dimensional sphere $S$ of radius $r$ in Euclidean $k$-space, $k \geqq 2$. Then

$$
L(C) \leqq r \kappa(C) .
$$

The proof of (3) again depends on the plane case and is motivated by the following construction. We form the cone $T$ over the curve $C$ with apex at the center of $S$, slit along a longest generator and develop the result in a plane. The resulting plane arc $C^{\prime}$ is completed to a closed plane curve $C^{\prime \prime}$ by attaching an arc of a circle. It is noted that the curvature of $C^{\prime}$ is equal pointwise to the geodesic curvature of $C$ with respect to $T$, which in turn is not greater, pointwise, than the curvature of $C$. The length of $C^{\prime}$ is the same as that of $C$. The inequality (2) applied to $C^{\prime \prime}$ now gives (3).

2. In this section we prove some lemmas which lead directly to the main theorem.

Lemma 1. Let $C$ be a rectifiable plane arc of length $L$. For any line $G$, let $n(p, \theta)$ be the number of intersections of $G$ with $C$, where $(p, \theta), p \geqq 0,0 \leqq \theta<2 \pi$, are the normal coordinates of $G$. Then

$$
L=\frac{1}{2} \int_{0}^{2 \pi} \int_{0}^{\infty} n(p, \theta) d p d \theta .
$$

Received March 9, 1961. 
This striking formula of Crofton is proved by Blaschke, [1], page 46.

LEMma 2. Let $C$ be a closed plane curve parametrized by arc length $s$. Let $\vec{r}=\vec{r}(s), 0 \leqq s \leqq L$, be the tracing vector of, $C$, and assume $\vec{r}^{\prime \prime}$ exists and is continuous except at a finite number of points $\vec{r}\left(s_{1}\right), \cdots, \vec{r}\left(s_{m}\right)$, where there are corners with "exterior" angles $\alpha_{1}, \cdots, \alpha_{m}$ respectively. Given any direction $\theta, 0 \leqq \theta<2 \pi$, let $n(\theta)$ be the number of tangents to $C$ orthogonal to that direction, where a tangent to $C$ at $\vec{r}\left(s_{i}\right), i=1,2, \cdots, m$, means a line through the point but not crossing $C$ at that point. Then

$$
\frac{1}{2} \int_{0}^{2 \pi} n(\theta) d \theta=\int\left|\vec{r}^{\prime \prime}(s)\right| d s+\sum_{i=1}^{m} \alpha_{i}=\text { total curvature of } C,
$$

where the integral on the right is extended over the smooth part of $C$.

Proof. We may write $n(\theta)=\sum_{i=0}^{m} n_{i}(\theta)$, where $n_{0}(\theta)$ counts the number of tangents to the smooth part of $C$ and $n_{i}(\theta), i \neq 0$, counts the number of tangents at $\vec{r}\left(s_{i}\right)$. Clearly $n_{i}$ takes only the values 0 or 1 , for $i \neq 0$, and

$$
\frac{1}{2} \int_{0}^{2 \pi} n_{i}(\theta) d \theta=\alpha_{i}, i \neq 0
$$

Finally, we have that

$$
\frac{1}{2} \int_{0}^{2 \pi} n_{0}(\theta) d \theta=\int\left|\vec{r}^{\prime \prime}(s)\right| d s,
$$

since the left hand side is just the measure of the spherical image (counting multiplicity) of the smooth part of $C$.

Lemma 3. Let $\vec{x}_{0}, \vec{x}_{1}, \cdots, \vec{x}_{n}$, be the successive vertices of a plane polygon $\bar{P}$ enclosed by a circle $S$ of radius $r_{0}$. Suppose further that the "initial" and "end" points, $\vec{x}_{0}$ and $\vec{x}_{n}$ respectively, lie on $S$. Let $\alpha_{i}, 0 \leqq \alpha_{i} \leqq \pi$, be the angle between $\vec{x}_{i+1}-\vec{x}_{i}$ and $\vec{x}_{i}-\vec{x}_{i-1}, i=1, \cdots$, $n-1$. If $\vec{x}_{0} \neq \vec{x}_{n}$, let $\alpha_{0}, 0 \leqq \alpha_{0} \leqq \pi$, be the angle between $\vec{x}_{1}-\vec{x}_{0}$ and the unit tangent vector to $S$ (with counterclockwise orientation) at $\vec{x}_{0}$, and let $\alpha_{n}, 0 \leqq \alpha_{n} \leqq \pi$, be the angle between $\vec{x}_{n}-\vec{x}_{n-1}$ and the unit tangent vector to $S$ (with counterclockwise orientation) at $\vec{x}_{n}$. If $\vec{x}_{0}=\vec{x}_{n}$, then simply let $\alpha_{0}\left(=\alpha_{n}\right), 0 \leqq \alpha_{0} \leqq \pi$, be the angle between $\vec{x}_{1}-\vec{x}_{0}$ and $\vec{x}_{0}-\vec{x}_{n-1}$. Let $L(\bar{P})$ be the length of $\bar{P}$.

Then if $\vec{x}_{0} \neq \vec{x}_{n}$, we have that

$$
L(\bar{P}) \leqq r_{0} \sum_{i=0}^{n} \alpha_{i} .
$$


If $\vec{x}_{0}=\vec{x}_{n}$, we have

$$
L(\bar{P}) \leqq r_{0} \sum_{i=0}^{n-1} \alpha_{i}
$$

(This lemma is a special case of Fáry's theorem for the plane. See [2], page 121. The proof we give here is essentially that of Fáry.)

Proof. We consider first the case where $\vec{x}_{0} \neq \vec{x}_{n}$. Let $\bar{S}$ be the are of $S$ traversed in a counterclockwise direction in going along $S$ from $\vec{x}_{n}$ to $\vec{x}_{0}$. Let $C=\bar{P} \cup \bar{S}$. Let $\delta$ be the angle subtended at the center of $S$ by $\bar{S}$. Then Lemma 1 gives,

$$
L(\bar{P})+r_{0} \delta=L(C)=\frac{1}{2} \int_{0}^{2 \pi} \int_{0}^{r_{0}} n(p, \theta) d p d \theta .
$$

It is easy to see, however, that $n(p, \theta) \leqq n(\theta)$ for $0 \leqq \theta<2 \pi$. Hence, by (9) and (5), we have

$$
L(\bar{P})+r_{0} \delta \leqq \frac{1}{2} r_{0} \int_{0}^{2 \pi} n(\theta) d \theta=r_{0}\left(\sum_{i=0}^{n} \alpha_{i}+\delta\right) .
$$

This gives the assertion for $\vec{x}_{0} \neq \vec{x}_{n}$. The case $\vec{x}_{0}=\vec{x}_{n}$ is now clear.

Lemma 4. Let $P$ be a closed polygon enclosed by a $k-1$ dimensional sphere $S$ of radius $r$ in Euclidean $k$-space. Let $\vec{y}_{0}, \vec{y}_{1}, \cdots, \vec{y}_{n}=\vec{y}_{0}$, be the successive vertices of $P$. Let $\beta_{i}, 0 \leqq \beta_{i} \leqq \pi$, be the angle between $\vec{y}_{i+1}-\vec{y}_{i}$ and $\vec{y}_{i}-\vec{y}_{i-1}, i=0,1, \cdots, n-1$, where $\vec{y}_{-1}$ is defined to be $\vec{y}_{n-1}$. Define the total curvature, $\kappa(P)$, of $P$, by

$$
\kappa(P)=\sum_{i=0}^{n-1} \beta_{i}, \quad(\text { See Milnor, [3], p. 249.) }
$$

Let $L(P)$ be the length of $P$. Then

$$
L(P) \leqq r \kappa(P) .
$$

Proof. Let $\vec{o}$ be the center of $S$. Assume that the vertices of $P$ are labeled so that $\vec{y}_{0}$ is no closer to $\vec{o}$ than any other vertex. Let $\beta_{i}^{\prime}$, $0 \leqq \beta_{i}^{\prime} \leqq \pi$, be the angle between $\vec{y}_{i}-\vec{o}$ and $\vec{y}_{i}-\vec{y}_{i+1}$; let $\beta_{i}^{\prime \prime}, 0 \leqq \beta_{i}^{\prime \prime} \leqq \pi$, be the angle between $\vec{y}_{i}-\vec{o}$ and $\vec{y}_{i}-\vec{y}_{i-1}, i=0,1, \cdots, n-1$. The triangle inequality applied to a spherical triangle cut out of a sphere centered at $\vec{y}_{i}$ shows that

$$
\beta_{i}^{\prime}+\beta_{i}^{\prime \prime} \geqq \pi-\beta_{i} \text {, and }\left(\pi-\beta_{i}^{\prime}\right)+\left(\pi-\beta_{i}^{\prime \prime}\right) \geqq \pi-\beta_{i} .
$$

Hence,

$$
\left|\pi-\left(\beta_{i}^{\prime}+\beta_{i}^{\prime \prime}\right)\right| \leqq \beta_{i}, \quad i=0,1, \cdots, n-1 .
$$


We now form the cone over $P$ with apex at $\vec{o}$, cut along the edge connecting $\vec{o}$ to $\vec{y}_{0}$ and develop the result in a plane as follows. Let $\vec{p}$ be a fixed point in the plane $R^{2}$. We map $\vec{y}_{0}$ into any point $\vec{x}_{0} \in R^{2}$ satisfying $\left|\vec{x}_{0}-\vec{p}\right|=\left|\vec{y}_{0}-\vec{o}\right|=r_{0}$. We next map $\vec{y}_{1}$ into a point $\vec{x}_{1} \in R^{2}$ satisfying $\left|\vec{x}_{1}-\vec{p}\right|=\left|\vec{y}_{1}-\vec{o}\right|=r_{1}$, and such that the angle $\delta_{1}$, from $\vec{x}_{0}-\vec{p}$ to $\vec{x}_{1}-\vec{p}$, measured in a counterclockwise direction, is equal to the angle $\delta_{1}, 0 \leqq \delta_{1} \leqq \pi$, between $\vec{y}_{0}-\vec{o}$ and $\vec{y}_{1}-\vec{o}$. In general we map $\vec{y}_{i}$ into $\vec{x}_{i} \in R^{2}$ so that $\left|\vec{x}_{i}-\vec{p}\right|=\left|\vec{y}_{i}-\vec{o}\right|=r_{i}$ and the angle $\delta_{i}$ from $\vec{x}_{i-1}-\vec{p}$ to $\vec{x}_{i}-\vec{p}$, measured counterclockwise, is equal to the angle $\delta_{i}, 0 \leqq \delta_{i} \leqq \pi$, between $\vec{y}_{i-1}-\vec{o}$ and $\vec{y}_{i}-\vec{o}$. This construction gives us a polygon $\bar{P}$ in $R^{2}$. Construct the circle $S^{\prime}$ of radius $r_{0}$ centered at $\vec{p}$. Then $\bar{P}$ is enclosed by $S^{\prime}$, and $\vec{x}_{0}$ and $\vec{x}_{n}$ (in general $\vec{x}_{0} \neq \vec{x}_{n}$ ) are on $S^{\prime}$. It is easily seen that the angle $\alpha_{i}, 0 \leqq \alpha_{i} \leqq \pi$, between $\vec{x}_{i}-\vec{x}_{i-1}$ and $\vec{x}_{i+1}-\vec{x}_{i}$, is $\left|\pi-\left(\beta_{i}^{\prime}+\beta_{i}^{\prime \prime}\right)\right|, i=1,2, \cdots, n-1$. It is also seen that the angles $\alpha_{0}$ and $\alpha_{n}$ described in Lemma 3 are equal to $(\pi / 2)-$ $\beta_{0}^{\prime}>0$ and $(\pi / 2)-\beta_{0}^{\prime \prime}>0$ respectively if $\vec{x}_{0} \neq \vec{x}_{n}$ and are both equal to $\pi-\left(\beta_{0}^{\prime}+\beta_{0}^{\prime \prime}\right)>0$ if $\vec{x}_{0}=\vec{x}_{n}$. Hence if $\vec{x}_{0} \neq \vec{x}_{n}$,

$$
\begin{aligned}
\sum_{i=0}^{n} \alpha_{i} & =\frac{\pi}{2}-\beta_{0}^{\prime}+\sum_{i=1}^{n-1}\left|\pi-\left(\beta_{i}^{\prime}+\beta_{i}^{\prime \prime}\right)\right|+\frac{\pi}{2}-\beta_{0}^{\prime \prime} \\
& =\sum_{i=0}^{n-1}\left|\pi-\left(\beta_{i}^{\prime}+\beta_{i}^{\prime \prime}\right)\right|,
\end{aligned}
$$

and if $\vec{x}_{0}=\vec{x}_{n}$,

$$
\sum_{i=0}^{n-1} \alpha_{i}=\sum_{i=0}^{n-1}\left|\pi-\left(\beta_{i}^{\prime}+\beta_{i}^{\prime \prime}\right)\right| .
$$

Therefore, by (8), $\left(8^{\prime}\right),(14)$, and $\left(14^{\prime}\right)$,

$$
L(P)=L(\bar{P}) \leqq r_{0} \sum_{i=0}^{n-1}\left|\pi-\left(\beta_{i}^{\prime}+\beta_{i}^{\prime \prime}\right)\right| \leqq r_{0} \sum_{i=0}^{n-1} \beta_{i}=r_{0} \kappa(P) \leqq r \kappa(P) .
$$

3. THEOREM 1. Let $C$ be a rectifiable closed curve enclosed by a $k-1$ dimensional sphere $S$ of radius $r$ in Euclidean $k$-space, $k \geqq 2$. Let $L(C)$ be the length of $C$ and $\kappa(C)$ be the total curvature of $C$. $(\kappa(C)=1 . u . b . \quad \kappa(P)$, where $P$ runs over all polygons inscribed in $C$. See Milnor, [3].) Then

$$
L(C) \leqq r \kappa(C)
$$

Proof. Given any $\varepsilon>0$, there is a polygon $P$ inscribed in $C$ such that $L(C)-L(P) \leqq \varepsilon$. We have that $\kappa(P) \leqq \kappa(C)$. Hence

$$
L(C)-\varepsilon \leqq L(P) \leqq r \kappa(P) \leqq r \kappa(C) .
$$

The theorem follows. 
CoRollary. Let $C$ be a closed curve of class $C^{\prime \prime}$ enclosed by a unit $k-1$ dimensional sphere in Euclidean k-space. Let $\kappa(s)=\left|\vec{r}^{\prime \prime}(s)\right|=$ curvature of $C$ at $\vec{r}(s), 0 \leqq s \leqq L(C)$. Then

$$
\max \kappa \geqq 1 \text {. }
$$

Proof.

$$
L(C) \leqq \kappa(C)=\int_{0}^{L(C)} \kappa(s) d s \leqq \max \kappa \cdot L(C) .
$$

Note that we have used the fact that the above integral form for the total curvature coincides with the previous definition. This is proved by Milnor in [3].

\section{REFERENCES}

1. W. Blaschke, Vorlesungen über Integralgeometrie, Chelsea, 1949.

2. I. Fáry, Sur certaines inégalités géométriques, Acta Sci. Math., Szeged, 12 (1950), 117-124.

3. J. W. Milnor, On the total curvature of knots, Ann. of Math., 52 (1950), 248-257. 



\section{PACIFIC JOURNAL OF MATHEMATICS}

\section{EDITORS}

Ralph S. Phillips

Stanford University

Stanford, California

M. G. Arsove

University of Washington

Seattle 5, Washington
A. L. Whiteman

University of Southern Californla

Los Angeles 7, California

Lowell J. Paige

University of California

Los Angeles 24, California

\section{ASSOCIATE EDITORS}

E. F. BECKENBACH

D. DERRY

H. L. ROYDEN

E. G. STRAUS

T. M. CHERRY

M. OHTSUKA

E. SPANIER

F. WOLF

\section{SUPPORTING INSTITUTIONS}

UNIVERSITY OF BRITISH COLUMBIA

STANFORD UNIVERSITY

CALIFORNIA INSTITUTE OF TECHNOLOGY

UNIVERSITY OF CALIFORNIA

MONTANA STATE UNIVERSITY

UNIVERSITY OF TOKYO

UNIVERSITY OF UTAH

UNIVERSITY OF NEVADA

NEW MEXICO STATE UNIVERSITY

OREGON STATE UNIVERSITY

UNIVERSITY OF OREGON

OSAKA UNIVERSITY

WASHINGTON STATE UNIVERSITY

UNIVERSITY OF WASHINGTON

UNIVERSITY OF SOUTHERN CALIFORNIA

AMERICAN MATHEMATICAL SOCIETY CALIFORNIA RESEARCH CORPORATION SPACE TECHNOLOGY LABORATORIES NAVAL ORDNANCE TEST STATION 


\section{Pacific Journal of Mathematics}

\section{Vol. 12, No. $1 \quad$ January, 1962}

Jonathan L. Alperin, Groups with finitely many automorphisms $\ldots \ldots \ldots \ldots \ldots \ldots \ldots \ldots$

Martin Arthur Arkowitz, The generalized Whitehead product ................ 7

John D. Baum, Instability and asymptoticity in toplogical dynamics . . . . . . . . . . 25

William Aaron Beyer, Hausdorff dimension of level sets of some Rademacher series .... $\quad 35$

Frank Herbert Brownell, III, A note on Cook's wave-matrix theorem . . . . . . . . . . . . . 47

Gulbank D. Chakerian, An inequality for closed space curves ................. 53

Inge Futtrup Christensen, Some further extensions of a theorem of Marcinkiewicz ....... 59

Charles Vernon Coffman, Linear differential equations on cones in Banach spaces . . . . . 69

Eckford Cohen, Arithmetical notes. III. Certain equally distributed sets of integers . . . . . 77

John Irving Derr and Angus E. Taylor, Operators of meromorphic type with multiple poles

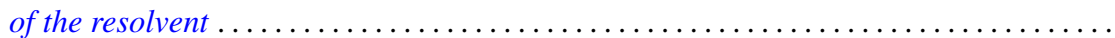

Jacob Feldman, On measurability of stochastic processes in products space .............

Robert S. Freeman, Closed extensions of the Laplace operator determined by a general class of boundary conditions, for unbounded regions ......................

Robert E. Fullerton, Geometric structure of absolute basis systems in a linear topological

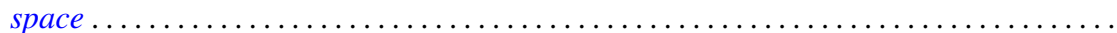

Dieter Gaier, On conformal mapping of nearly circular regions

Andrew Mattei Gleason and Hassler Whitney, The extension of linear functionals defined

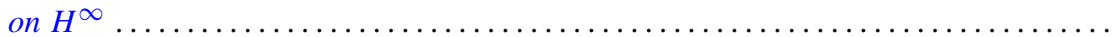

Seymour Goldberg, Closed linear operators and associated continuous linear

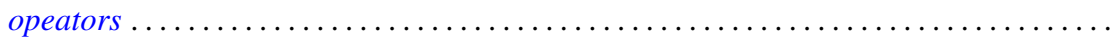

Basil Gordon, Aviezri Siegmund Fraenkel and Ernst Gabor Straus, On the determination of sets by the sets of sums of a certain order

Branko Grünbaum, The dimension of intersections of convex sets. .

Paul Daniel Hill, On the number of pure subgroups

Robert Peter Holten, Generalized Goursat problem . .

Alfred Horn, Eigenvalues of sums of Hermitian matrices ...........

Henry C. Howard, Oscillation and nonoscillation criteria for

$$
y^{\prime \prime}(x)+f(y(x)) p(x)=0
$$

Taqdir Husain, $S$-spaces and the open mapping theorem ...

Richard Eugene Isaac, Markov processes and unique stationary probability measures ...

John Rolfe Isbell, Supercomplete spaces ....................

John Rolfe Isbell, On finite-dimensional uniform spaces. II .........

N. Jacobson, A note on automorphisms of Lie algebras ..............

Antoni A. Kosinski, A theorem on families of acyclic sets and its applications

Marvin David Marcus and H. Minc, The invariance of symmetric functions of singular values...

Ralph David McWilliams, A note on weak sequential convergence.

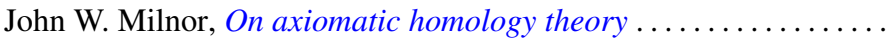

Victor Julius Mizel and Malempati Madhusudana Rao, Nonsymmetric projections in

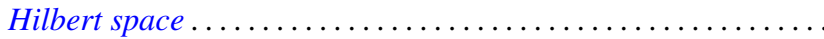

Calvin Cooper Moore, On the Frobenius reciprocity theorem for locally compact

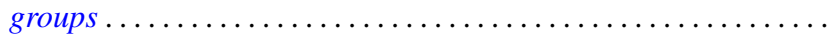

Donald J. Newman, The Gibbs phenomenon for Hausdorff means . 